\title{
A new technique for delivering a polyglycolic acid sheet to cover a large mucosal defect: the Swiss roll method
}

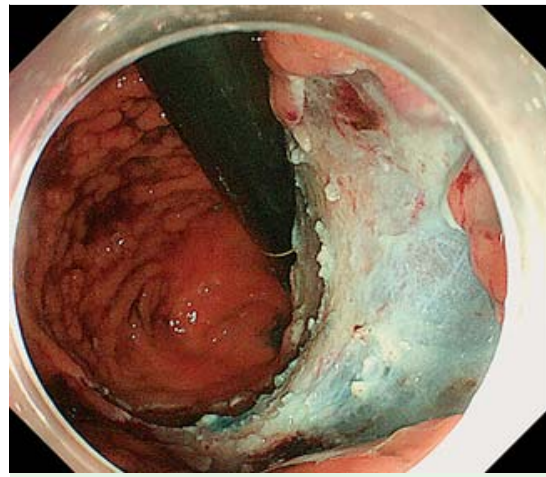

Fig. 1 A large artificial ulcer was present after piecemeal endoscopic mucosal resection.

Endoscopic tissue shielding with polyglycolic acid (PGA) sheets (Neoveil; Gunze Co., Kyoto, Japan) and fibrin glue (Beriplast P CombiSet; CSL Behring Pharma, Tokyo, Japan) is a promising method for preventing postoperative complications [1-3]. However, this technique is thought to be inefficient for covering large mucosal defects because it takes time to deliver many small PGA sheets. Accordingly, we devised and implemented a new technique involving the delivery of a single large PGA sheet.

A 25-year-old woman with familial adenomatous polyposis was treated using a standard-caliber endoscope by rectal piecemeal endoscopic mucosal resection, leaving a large mucosal defect ( $\bullet$ Fig.1). For our new technique, we used a PGA sheet and its accompanying plastic packaging sheet. We cut the PGA sheet to cover the artificial ulcer, and then we cut the plastic sheet to a slightly smaller size. We attached one edge of the plastic sheet to a small-caliber endoscope with tape. Next, we placed the PGA sheet over the plastic sheet, and we proceeded to roll the two layers around the small-caliber endoscope like a Swiss roll, making sure the PGA sheet was on the outside. We placed the enwrapped small-caliber endoscope inside an overtube (Flexible Overtube; Sumitomo Bakelite Inc., Tokyo, Japan) to prevent contact with any moisture.

We delivered the second endoscope within the overtube to the ulcer and then removed the overtube. Using the biopsy
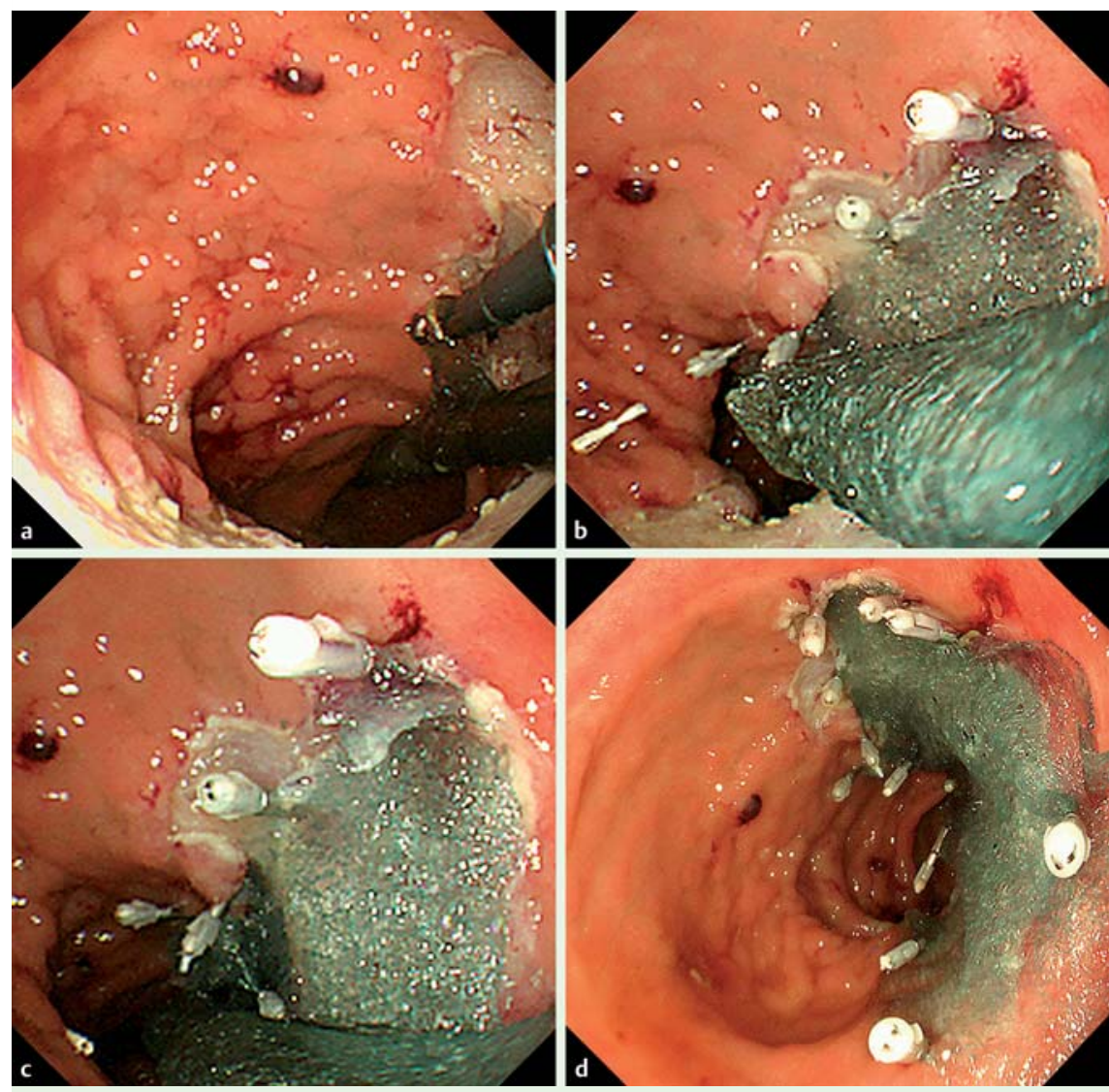

Fig. 2 Endoscopic view showing the Swiss roll method for application of a polyglycolic acid (PGA) sheet to cover an artificial ulcer. a One end of the PGA sheet was grasped using the main endoscope's biopsy forceps. $\mathbf{b}$ The end of the PGA sheet was fixed to one edge of the ulcer with clips. $\mathbf{c}$ The PGA sheet was unrolled over the ulcer. $\mathbf{d}$ The artificial ulcer was covered with a single large PGA sheet, which was fixed to the remaining edges of the ulcer using several clips.

forceps of the standard-caliber endoscope, we grasped one end of the PGA sheet ( $\nabla$ Fig. 2 a) and fixed it to one edge of the ulcer with clips ( Fig. $2 \mathbf{b}$ ). We proceeded to unroll the PGA sheet over the ulcer $($ Fig. $\mathbf{2 c}$ ). The plastic sheet underneath gradually separated from the PGA sheet and remained curled around the small-caliber endoscope. We then fixed the PGA sheet using several clips $(\checkmark$ Fig. 2d). This technique can be used to achieve the delivery of a single large PGA sheet to cover a sizeable mucosal defect.

Endoscopy_UCTN_Code_TTT_1AQ_2AZ

Competing interests: None

\section{Kunihiro Tsuji, Hisashi Doyama, Hiroyoshi Nakanishi, Kazuhiro Matsunaga, Shinya Yamada}

Department of Gastroenterology, Ishikawa Prefectural Central Hospital, Kanazawa, Japan

\section{References}

1 Takimoto K, Toyonaga T, Matsuyama K. Endoscopic tissue shielding to prevent delayed perforation associated with endoscopic submucosal dissection for duodenal neoplasms. Endoscopy 2012; 44: E414-E415

2 Tsuji Y, Ohata K, Gunji T et al. Endoscopic tissue shielding method with polyglycolic acid sheets and fibrin glue to cover wounds after colorectal endoscopic submucosal dissection (with video). Gastrointest Endosc 2014; 79: 151 - 155 
3 Ono S, Tsuji Y, Fujishiro $M$ et al. An effective technique for delivery of polyglycolic acid sheet after endoscopic submucosal dissection of the esophagus: the clip and pull method. Endoscopy 2014; 46: E44-E45

\section{Bibliography}

Dol http://dx.doi.org/

10.1055/s-0034-1377767

Endoscopy 2014; 46: E547-E548

(c) Georg Thieme Verlag KG

Stuttgart · New York

ISSN 0013-726X

\section{Corresponding author}

\section{Kunihiro Tsuji, MD}

Department of Gastroenterology Ishikawa Prefectural Central Hospital 2-1 Kuratsuki-higashi, Kanazawa Ishikawa 920-8530

Japan

Fax: +81-76-2385366

kt98052@yahoo.co.jp 